\title{
Role of trefoil factor 1 in gastric cancer and relationship between trefoil factor 1 and gastrokine 1
}

\author{
WEI MAO ${ }^{1,3^{*}}$, JIE CHEN $^{1 *}$, TIE-LI PENG ${ }^{1}$, XIAO-FEI YIN ${ }^{1}$, LIAN-ZHOU CHEN $^{2}$ and MIN-HU CHEN ${ }^{1}$ \\ ${ }^{1}$ Department of Gastroenterology, ${ }^{2}$ Tumor Tissue Bank, The First Affiliated Hospital of Sun Yat-Sen University; \\ ${ }^{3}$ Department of Gastroenterology, The Third Affiliated Hospital of Sun Yat-Sen University, Guangzhou, P.R. China
}

Received April 23, 2012; Accepted June 18, 2012

DOI: 10.3892/or.2012.1939

\begin{abstract}
Trefoil factor 1 (TFF1) is a small cysteine-rich secreted protein which is principally expressed in the superficial cells of gastric mucosa. In gastric cancer, TFF1 is downregulated and plays an important role. Gastrokine 1 (GKN1) is a secreted protein with similar expression and biological functions to TFF1. This study aimed to determine the expression and biological functions of TFF1 and the relationships between TFF1 and GKN1 in gastric cancer. RT-PCR and immunohistochemistry were performed to detect TFF1 expression in gastric cancer cell lines and tissues. The transfected and co-transfected AGS cells which stably expressed TFF1 or both TFF1 and GKN1 were generated. Phenotypic changes such as cell viability, apoptosis and cell cycle modulation were assayed in the transfected cells. We found that TFF1 expression was significantly downregulated or lost in gastric cancer cell lines, gastric dysplasia and cancer. Restoration of TFF1 expression in AGS cells suppressed tumor cell viability and arrested AGS cells in the G1-S transition phase after olomoucine treatment. However, TFF1 was unable to induce cell apoptosis. In co-transfected cells, we found that TFF1 and GKN1 did not directly interact at the protein level. GKN1 was unable to cooperate with TFF1 on cell viability suppression, cell apoptosis and differentiation. Together, these results indicate that TFF1 expression is significantly downregulated in gastric cancer. TFF1 inhibited cell proliferation by delaying G1-S phase transition but not by inducing apoptosis. TFF1 may not interact or cooperate with GKN1 at the protein and functional level.
\end{abstract}

\section{Introduction}

Gastric cancer is the second most common cause of cancer mortality worldwide (1-3). Although the molecular mechanisms of gastric cancer are still unclear, some molecular changes

Correspondence to: Dr M.H. Chen, Department of Gastroenterology, The First Affiliated Hospital of Sun Yat-Sen University, 58 Zhongshan II Road, Guangzhou 510080, P.R. China

E-mail: chenminhu@vip.163.com

*Contributed equally

Key words: trefoil factor 1, gastrokine 1, gastric cancer, apoptosis, cell cycle in the development of tumor were characterized. In recent studies, TFF1 is a spotlight molecule in gastric cancer. TFF1 is a 60 -amino acid, highly conserved protein with a clover leaf-like structure formed by cysteine disulphide bonds and belongs to the trefoil factor family (4-6). TFF1 was found abundantly expressed in normal gastric mucosa. As a secreted protein, TFF1 protein was synthesized and secreted by the gastric epithelial cells and expressed in the upper part of the pits. In ulceration or inflammatory diseases, TFF1 induced epithelial restitution after injury and protects the integrity of the epithelial barrier (7-10). TFF1 was also involved in gastric carcinogenesis. In most gastric adenocarcinomas, the expression of TFF1 was downregulated or absent comparing to abundant in normal gastric mucosa $(11,12)$. A previous study found that the TFF1-deficient mice were dysfunctional and showed severe hyperplasia and dysplasia, and all homozygous mutant mice developed antropyloric adenoma (13). Moreover, TFF1 demonstrated inhibition of cell growth and arrest of cell difference in gastric cancer $(14,15)$. Thus, TFF1 is hypothesized as a tumor suppressor in gastric cancer. TFF1 was also involved in other human cancers such as breast, pancreas and prostate cancer, but TFF1 stimulates cancer cell poliferation and migration in these cancers (16-18). Since the biological effects are not clear in different cancers, the role of TFF1 should be further addressed in gastric cancer.

GKN1, which contains a conserved BRICHOS domain, is a secreted protein belonging to gastrokine family (19). It is abundantly expressed in gastric epithelium $(19,20)$. GKN1 plays a protective role in gastric mucosa but is downregulated in gastric cancer $(21,22)$. In other words, GKN1 and TFF1 were similar in both the expressed location and physical functions. GKN2, a number of gastrokine family, also contains a BRICHOS domain and is a downstream gene of GKN1 (23). Several studies found that GKN2 combined with TFF1 as a heterodimer to maintain the stabilization of mucosa (24-26). GKN1 and GKN2 showed remarkable similarity in protein level (24). Thus, it is reasonable to speculate that there may be some relationships between GKN1 and TFF1 in protein or functional level.

In the present study, we investigated the role of TFF1 and the relationships between TFF1 and GKN1 in gastric cancer.

\section{Materials and methods}

Tissue specimens. Normal gastric mucosa tissues were collected from 20 recruited healthy volunteers by endoscopy biopsy. 
Tissues of gastric dysplasia were collected from 40 patients by endoscopy biopsy. And tissues of gastric tumors and their corresponding adjacent non-tumor tissues were collected from 39 gastric cancer patients who underwent surgery (Table I). None of the gastric cancer patients received preoperative chemotherapy or radiotherapy. Written informed consent was signed by all participants, and the research was approved by our Institutional Review Board. All tissue specimens were diagnosed by pathologists and confirmed by an experienced pathologist (27).

Gastric cancer cell lines. Seven gastric cancer cell lines, MKN28, MKN45, AGS, NCI-N-87, SNU 1, SNU16 and KATO, were obtained from the Riken Cell Bank (Tsukuba, Japan) or the American Type Culture Collection (Manassas, VA, USA). Cells were cultured in RPMI-1640 medium containing $10 \%$ fetal bovine serum (Hyclone, Logan, USA), and maintained at $37^{\circ} \mathrm{C}$ in a humidified $5 \% \mathrm{CO}_{2}$ atmosphere.

RNA isolation and RT-PCR. Gastric cancer tissue specimens and gastric cancer cell lines were homogenized with an ultrasound homogenizer. Total RNA from tissues and tumor cells was isolated using the Qiagen RNeasy Mini Kit(Qiagen, Hilden, Germany) according to the manufacturer's instructions. After quantification, RNA was reverse transcribed into cDNA using ReverTra Ace ${ }^{\mathrm{TM}}$ Kit (Toyobo, Osaka, Japan). The newly synthesized cDNA was then amplified by PCR with specific primers for the TFF1 gene (5'-CACCATGGAGAACAAGGTGA-3' and 5'-GGGACGTCGATGGTATTAGG-3') or $\beta$-actin. PCR products were subsequently electrophoresed on a $1.5 \%$ agarose gel.

Immunohistochemistry. Paraffin sections (4 $\mu \mathrm{m}$ thick) were prepared. In short, paraffin sections were deparaffinised and rehydrated. After antigen retrieval with citrate, endogenous peroxidase was quenched with $\mathrm{H}_{2} \mathrm{O}_{2}$. The sections were then incubated with mouse anti-human TFF1 (1:200) (Abnova, Taipei, R.O.C.) at room temperature for $2 \mathrm{~h}$. Then, a 2 -step detection method was used according to the manufacturer's instructions (Envision $^{\mathrm{TM}}$ Detection kit, Gene Tech, Shanghai, China) by incubation of the tissue with the ChemMate ${ }^{\mathrm{TM}}$ EnVision $^{\mathrm{TM}} / \mathrm{HRP}$ for $30 \mathrm{~min}$ at room temperature. The reaction was visualized by the CheMate ${ }^{\mathrm{TM}}$ DAB plus chromogen. Lastly, the sections were counterstained with hematoxylin solution. Negative controls were performed by staining with PBS. Immunostaining was assessed by an experienced pathologist who was blinded to the clinical data of the patients.

Construction of stable gene transfection. TFF1 and GKN1 cDNA was amplified from total RNA of the normal gastric mucosa using PCR. GKN1 CDS fragments with SalI and $B a m \mathrm{HI}$ restriction sites and TFF1 CDS fragments with KpnI and $B g l I$ restriction sites were then solely or simultaneously inserted into the pBudCE4.1 vector (Invitrogen, Carlsbad, CA, USA), which contains two promoters for independent expression of two recombinant proteins, using a DNA ligation kit (Takara, Dalian, China). After transformation into DH5a E. coli competent cells, the plasmid was amplified and the contructs were verified by sequencing. To generate gastric cancer cells expressing TFF1 or co-expressing TFF1 and GKN1 (TFF1-GKN1), gastric cancer AGS cells were grown to 50-75\% confluency in a 6-well
Table I. Study population.

\begin{tabular}{|c|c|c|c|c|}
\hline \multirow{2}{*}{ Histological type } & \multirow{2}{*}{$\begin{array}{l}\text { No. of } \\
\text { patients }\end{array}$} & \multicolumn{2}{|c|}{ Gender } & \multirow{2}{*}{$\begin{array}{l}\text { Age (years) } \\
\text { Mean } \pm \text { SD }\end{array}$} \\
\hline & & Male & Female & \\
\hline Normal gastric mucosa & 20 & 10 & 10 & $44.6 \pm 12.7$ \\
\hline Gastric dysplasia & 40 & 30 & 10 & $64.0 \pm 11.4$ \\
\hline Gastric cancer & 39 & 23 & 16 & $53.0 \pm 10.0$ \\
\hline
\end{tabular}

plate, and subjected to the Lipofectamine-mediated transfection (Invitrogen) according to the manufacturer's protocol. The TFF1 and TFF1-GKN1 transfected gastric cancer cells were then selected in medium containing Zeocin (Invitrogen). After the transfected cells formed individual cell colonies, stable cells were obtained and then confirmed for TFF1 or GKN1 expression by RT-PCR, western blotting and immunoblot analyses. Vector was also transfected into AGS cells as control cells.

Co-IP and western blotting. Total cellular protein was extracted from normal gastric mucosa tissues, control cells and TFF1-GKN1 co-transfected cells using a lysis buffer containing protease inhibitor cocktail (Roche, Mannheim, Germany). An equal amount of protein $(500 \mu \mathrm{g})$ was incubated with a 1:2001:500 dilution of anti-TFF1 antibody for $1.5 \mathrm{~h}$ at $4^{\circ} \mathrm{C}$ followed by addition of protein A/G Plus agarose beads (Santa Cruz Biotechnology, Santa Cruz, CA, USA) and incubation for an additional $1.5 \mathrm{~h}$ at the same conditions. The protein/antibody/ beads mix was then washed three times with PBS buffer, resuspended in SDS sample buffer, and denatured at $95^{\circ} \mathrm{C}$ for $5 \mathrm{~min}$. All samples were resolved by $10 \%$ SDS-PAGE, and electroblotted onto PVDF membranes. Membranes were blocked in 5\% non-fat milk for $2 \mathrm{~h}$, and then incubated for $2 \mathrm{~h}$ with a 1:500 dilution of anti-GKN1 antibody (Abnova). After washed with PBS buffer three times and incubation for $2 \mathrm{~h}$ with the appropriate secondary antibody, enhanced chemiluminescence (Pierce, Rockford, IL, USA) was used for protein visualization.

Cell viability (MTT) assay. To detect changes in tumor cell viability after TFF1 or TFF1-GKN1 transfection, a total of $1 \times 10^{4}$ trypsin-dispersed cells was seeded into each well of a 96-well plate, and cultured for 24 or $48 \mathrm{~h}$. Next, $20 \mu \mathrm{l}$ of MTT (5 g/l) was added to each well and incubated for additional $4 \mathrm{~h}$ at $37^{\circ} \mathrm{C}$. Culture medium was then replaced with $200 \mu \mathrm{l}$ of dimethyl sulfoxide (DMSO) and the absorbance rate was determined using an ELISA reader at $490 \mathrm{~nm}$. Cell growth inhibition rate was calculated as (the value of experimental group OD/the value of control group OD) x $100 \%$.

Annexin V apoptosis assay. To detect tumor cell apoptosis, the TFF1 or TFF1-GKN1 transfected AGS cells were seeded into 60-mm diameter culture plates, and cultured for 24 and $48 \mathrm{~h}$. The apoptotic rates were analyzed by flow cytometry using an annexin V-FITC/PI kit (Keygen, Nanjing, China). Staining was performed according to the manufacturer's instructions, and flow cytometry was conducted with a flow cytometer (BeckmanCoulter, Brea, CA, USA). Cells with annexin V (-) and PI (-) were deemed viable cells. Cells with annexin V (+) and PI (-) were 
A

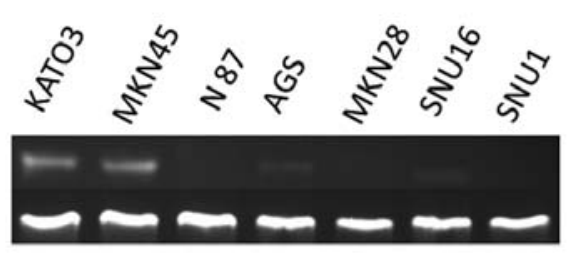

B

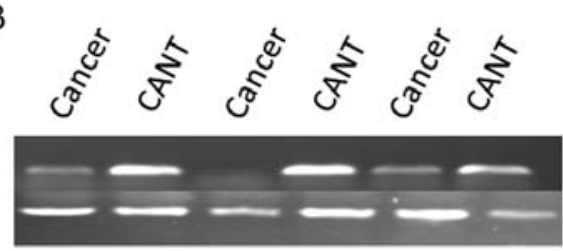

Figure 1. mRNA expression of TFF1 in gastric cancer cell lines and gastric tissue specimens. (A) Gastric cancer cell lines. TFF1 mRNA expression was low in gastric cancer KATO3, MKN45, still lower expressed in AGS and SNU16 cells, and was lost in N87, MKN28 and SNU1 cells. (B) Gastric tissue specimens. Expression of TFF1 mRNA was significantly downregulated or even absent in gastric cancer tissues but abundant in their corresponding adjacent non-cancer tissues (CANT).
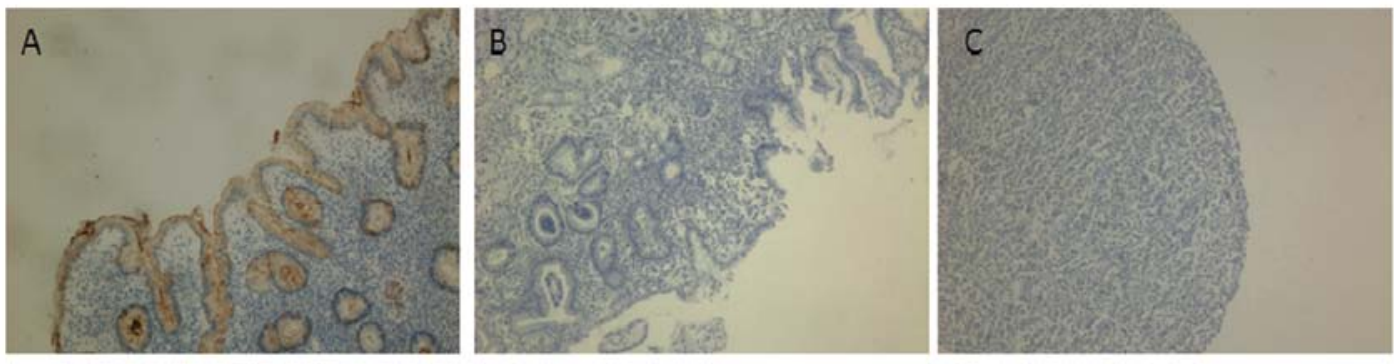

Figure 2. Immunohistochemical detection of TFF1 protein in gastric tissue specimens. TFF1 protein was abundant in normal gastric mucosa, but downregulated or even absent in gastric dysplasia and cancer. (A) Normal gastric mucosa. (B) Gastric dysplasia tissue. (C) Gastric cancer tissue.

deemed early apoptotic cells. Cells with both annexin V (+) and PI (+) were deemed late apoptotic cells.

Cell cycle analysis. To analyze cell cycle distribution, transfected cells were grown and treated with $25 \mu \mathrm{M}$ molomoucine (Santa Cruz) for $1 \mathrm{~h}$, and then incubated in regular culture medium without molomoucine for an additional $1 \mathrm{~h}(15)$. Cells were then collected and subjected to cell cycle analysis by flow cytometry.

Statistical analysis. All quantitative data were expressed as mean \pm SD and analyzed by Student's t-tests. The differential expression of TFF1 among different groups was determined by Kruskal-Wallis test. All statistical analyses were performed using the SPSS statistical software package (version 11.0, SPSS Inc. Chicago, IL, USA). P $<0.05$ was considered statistically significant.

\section{Results}

Expression of TFF1 in cancer cell lines and gastric tissue specimens. Results of RT-PCR analysis showed that TFF1 mRNA had low expression in gastric cancer KATO3, MKN45, lower expressed in AGS and SNU16 cells, and was lost in N87, MKN28 and SNU1 cells (Fig. 1A). In 39 gastric cancer tissues, TFF1 mRNA was only weakly expressed in 15 tissue specimens and absent in the remaining 24 tissues (Fig. 1B). However, TFF1 mRNA was abundant in all of the 39 corresponding adjacent non-cancer tissues as well as in normal gastric epithelial cells obtained from 20 healthy volunteers.

Next, we immunohistochemically stained TFF1 in the tissue sections from patients. We found that TFF1 protein expression was significantly downregulated or even lost in dysplastic and cancer tissues specimens. In contrasts, the TFF1 protein was
Table II. TFF1 expression detected by immunohistochemistry in gastric tissues.

\begin{tabular}{lcrrrrrr}
\hline Histological type & $\begin{array}{c}\text { No. of } \\
\text { patients }\end{array}$ & - & + & ++ & +++ & P-value \\
\hline Normal gastric mucosa & 20 & 0 & 0 & 0 & 20 & \\
Dysplastic lesion & 40 & 22 & 18 & 0 & 0 & $<0.05$ \\
Gastric cancer & 39 & 27 & 12 & 0 & 0 & $<0.05$ \\
\hline
\end{tabular}

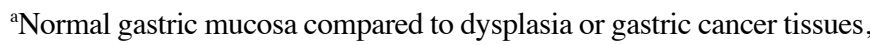
$\mathrm{P}<0.05$.

strongly expressed in the normal mucosa (Fig. 2). This reduction in protein expression was statistically significant $(\mathrm{P}<0.05)$ (Table II).

Identification of TFFI and GKN1 protein interaction by Co-IP. To determined protein interaction between TFF1 and GKN1, we successfully generated AGS cells that stably co-expressed TFF1 and GKN1; expression was confirmed by RT-PCR, western blotting and immunohistochemistry. In cells, both GKN1 and TFF1 showed granular cytoplasmic distribution. In normal gastric mucosa and co-transfected AGS cells, the protein interaction between GKN1 and TFF1 were investigated by Co-IP analysis. The negative results of Co-IP demonstrate that there was no direct molecular interaction between GKN1 and TFF1 in protein level.

Effect of TFF1 or TFF1-GKN1 on AGS cell proliferation. In the previous experiments, we confirmed TFF1 was downregulated in gastric cancer. Then effects of TFF1 on cancer cells were 


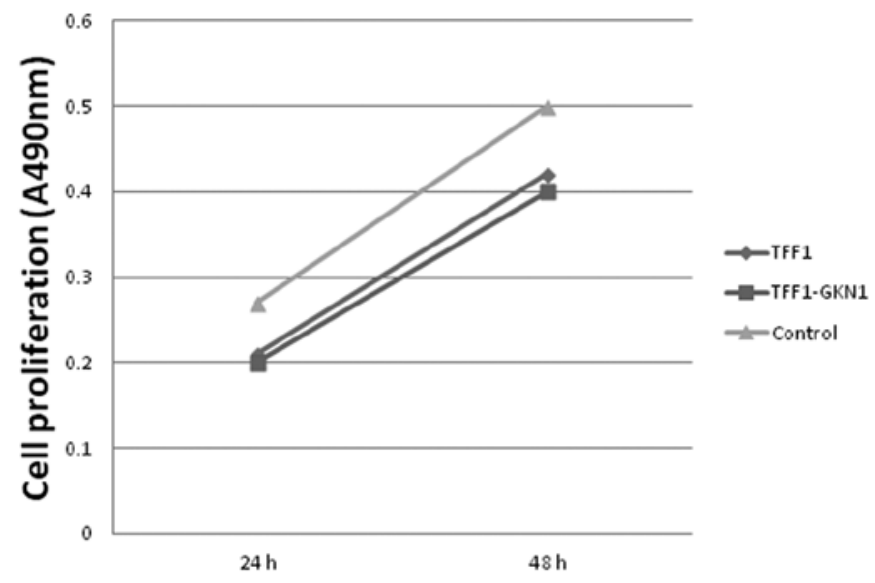

Figure 3. Effect of TFF1 or TFF1-GKN1 on AGS cells proliferation. The proliferation of TFF1 transfected cells was significantly decreased comparing to the control cells in both $24-$ and 48 -h cultures $(\mathrm{P}<0.01)$. But the cell proliferation was not statistically significant compared between TFF1 transfected cells and TFF1-GKN1 co-transfected cells $(\mathrm{P}>0.05)$

determined. The MTT results showed that TFF1 significantly decreased the proliferation of AGS cells as compared to the vector-transfected cells in both $24-$ and 48 -h cultures $(\mathrm{P}<0.05)$, while the proliferation between the TFF1 transfected cells and the TFF1-GKN1 co-transfected cells was not statistically significant in either 24-or 48-h cultures ( $\mathrm{P}>0.05)$ (Fig. 3).

Effect of TFF1 and TFF1-GKN1 on AGS cell apoptosis. The cell apoptosis of the transfected cells was also investigated. The flow cytometry results demonstrated that cell apoptosis rate was not statistically significant between TFF1 transfected cells $(2.73 \pm 0.30 \%)$ and vector-transfected cells $(2.76 \pm 0.38 \%)$ in $24 \mathrm{~h}$ ( $\mathrm{P}>0.05)$, while TFF1-GKN1 co-transfected cells showed an increased apoptosis rate $(4.61 \pm 0.42 \%)$ comparing to TFF1 transfected cells or vector-transfected cells $(\mathrm{P}<0.05)$ (Fig. 4).

Cell cycle redistribution of transfected cells. We then investigated the effects of TFF1 and TFF1-GKN1 on cell cycle redistribution. Olomoucine, a purine derivative and a cyclin-dependent kinase (CDK) inhibitor, was used to enrich parental AGS cells in the G1 phase. Specifically, cells were arrested in the cell cycle by
$1 \mathrm{~h}$ olomoucine treatment and continued to be incubated for another $1 \mathrm{~h}$ without olomoucine. The cell cycle distribution of TFF1 transfected cells changed from (G1 40.4\%, S 36.1\%) to (G1 57.0\%, S 21.7\%). The cell cycle distribution of TFF1-GKN1 co-transfected cells changed from (G1 42.7\%, S 37.0\%) to (G1 45.8\%, S 28.5\%) (Fig. 5). These data demonstrated that TFF1 was superior to TFF1-GKN1 in arresting AGS cells in the G1-S transition phase.

\section{Discussion}

In the present study, we have shown that TFF1 mRNA was downregulated or even absent in gastric cancer cell lines, gastric dysplasia and cancer tissues, but TFF1 protein was abundant in the corresponding adjacent non-cancer tissues as well as in normal gastric mucosa. These results suggested that downregulation of TFF1 is a frequent event in gastric precancer or cancer, and the presence of TFF1 may protect gastric mucosa from neoplasia.

The effects of TFF1 on gastric cancer cells were investigated. Our MTT data showed that endogenous TFF1 protein reduced cell proliferation. It was consistent with the result of a previous study that exogenous TFF1 protein could reduce gastric cancer cell proliferation in a dose-dependent manner (14). The following data of flow cytometry assay showed that TFF1 was unable to induce apoptosis in cancer cells, but TFF1 participated in cell differentiation by delaying G1-S cell phase transition. It indicated that the ability of cell viability suppression was due to the regulation of cell differentiation but not the induction of apoptosis. These data were consistent with the previous studies $(14,15,28)$. Taken together, the results suggested that TFF1 may play an important role as a tumor suppressor in gastric cancer.

The protein interaction and functional relationships between TFF1 and GKN1 were further addressed. We successfully cloned and co-transfected TFF1 and GKN1 into gastric cancer AGS cells and the cells stably expressed both TFF1 and GKN1 protein. Both TFF1 and GKN1 showed granular cytoplasmic distribution in the the co-transfected cells. The expression locations of TFF1 and GKN1 in gastric mucosa are similar. In gastric mucosa, GKN1 plays an important role in maintaining mucosal integrity and mediating repair after injury. In gastric cancer, GKN1 showed downregulated expression and inhibited cell growth. In view of the similar expression and biological

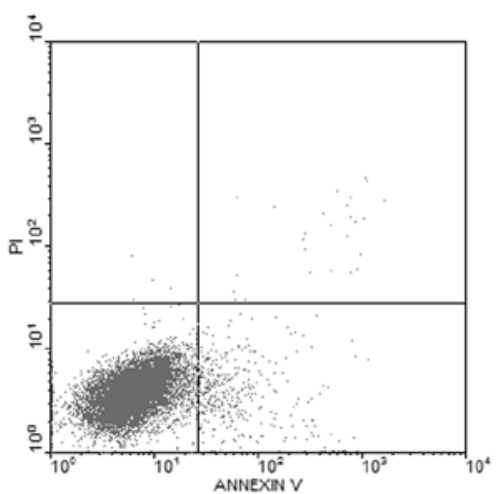

Control cells

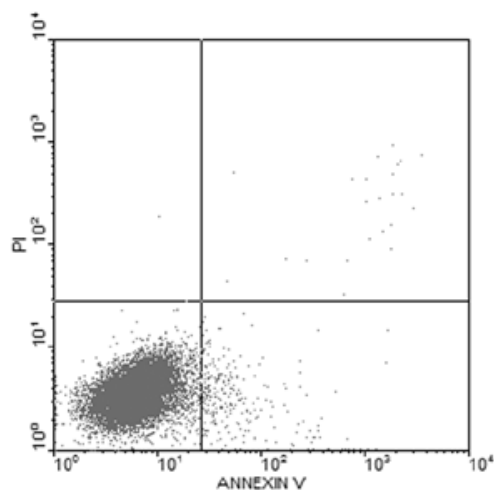

TFF1 tranfected cells

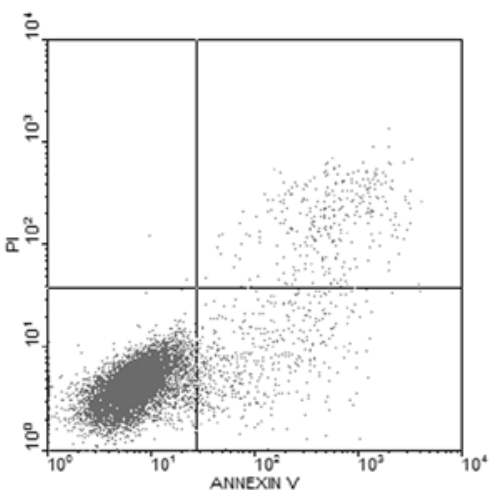

TFF1-GKN1 co-transfected cells

Figure 4. The apoptosis rate of TFF1 and TFF1-GKN1 transfected AGS cells. Cell apoptosis rate was not statistically significant between TFF1 transfected cells and vector-transfected cells in $24 \mathrm{~h}$ (P>0.05), while TFF1-GKN1 co-transfected cells showed an increased apoptosis rate compared to TFF1 transfected cells or vector-transfected cells $(\mathrm{P}<0.05)$. 
A

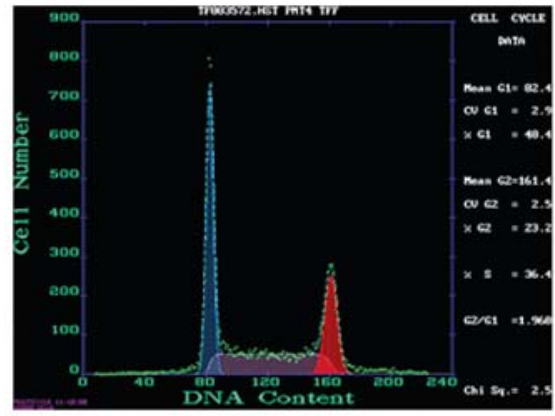

TFF1 transfected cells

B

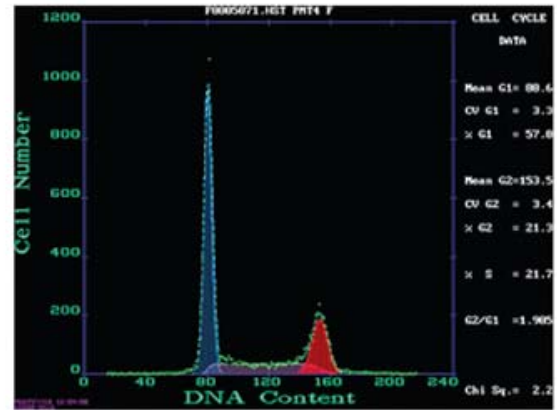

TFF1 transfected cells

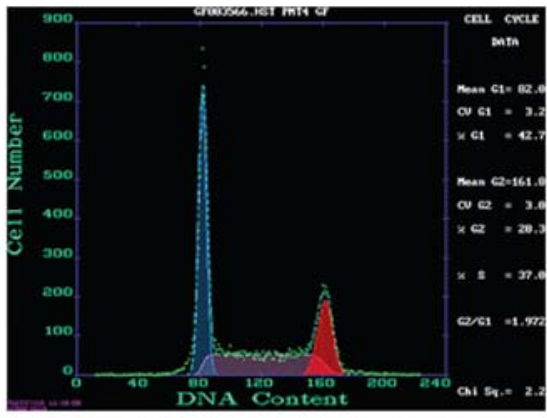

TFF1-GKN1 co-transfected cells

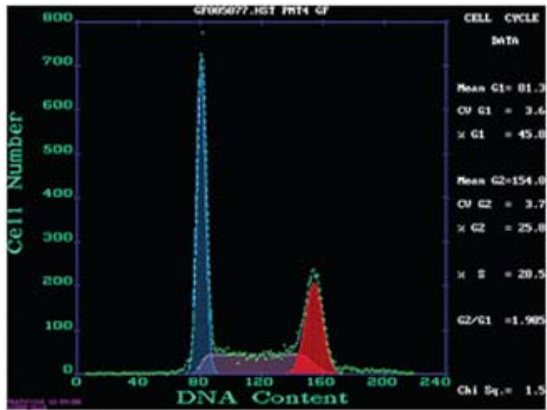

TFF1-GKN1 co-transfected cells

Figure 5. Cell cycle redistribution of transfected cells. TFF1 may arrest AGS cells in the G1-S transition phase. TFF1 was superior to TFF1-GKN1 in arresting AGS cells in the G1-S transition phase. (A) Cell cycle distribution by 1-h olomoucine treatment. (B) Cell cycle redistribution after an additional 1-h incubation without olomoucine.

functions, it was reasonable to speculate that there is some possible relationships between TFF1 and GKN1. GKN2 is a downstream gene of GKN1 and belongs to gastroinkase family (23). Recent studies have shown that GKN2 may interact with TFF1 to be a heterodimer. The ligament between GKN2 and TFF1 in the heterodimer is an intermolecular disulfide bond between cysteine residues in the carboxy-terminus of TFF1 and in the BRICHOS domain of GKN2 $(24,26,29,30)$. However, the relationship between TFF1 and GKN1 has not been reported. To determine whether TFF1 conjoin to GKN1 by a biochemistry connection between these two molecules, we used Co-IP assay to detect the TFF1-GKN1 co-transfected AGS cells and normal gastric tissues which all abundantly expressed both TFF1 and GKN1 protein. The negative result of Co-IP identified that there was no directly protein-protein interactions between TFF1 and GKN1 to be a protein complex. GKN1 and GKN2 have the same intron and exon structures, and are located in upstream and downstream on the same chromosomes of genomes. GKN1 has a significant homology to GKN2 in protein level (24). Specially, both GKN1 and GKN2 contain the conserved BRICHOS domain which contains a pair of conserved cysteine residues (19). The most striking difference between GKN2 and GKN1 was that GKN2 contains an additional cysteine residue at position 38, which locates within the BRICHOS domain. Thus, this additional cysteine residue of GKN2 interacted with the cysteine 58 of TFF1 in the carboxy-terminus to form intermolecular disulfide bonds and stabilizing the interaction between the two molecules (GKN2 and TFF1). GKN1 does not have this addi- tional cysteine residue, therefore GKN1 was unable to interact with TFF1 in protein level.

Although TFF1 did not interact with GKN1 to form a heterodimer, the expression and biological functions in gastric cancer of these two molecules were similar. We hypothesized that these two molecules may cooperate or perform synergistic effects in gastric cancer, but TFF1-GKN1 did not show more cells viability inhibition than TFF1 by MTT assay. GKN1 was demonstrated apoptosis induction in gastric cancer cells in a previous study (31). Our results show that TFF1 participated in cell differentiation by delaying G1-S phase transition. The co-transfected cells with presence of TFF1 and GKN1 were observed with a lower rate in holding cells in the G1-S transition phase compared to TFF1 transfected cells. In normal gastric mucosa, GKN1 was mitogenic and motogenic for facilitating restitution and proliferation after gastric mucosal injury. Thus, GKN1 may weaken the effect of TFF1 in delaying G1-S phase transition in the co-transfected cells.

In conclusion, TFF1 may be a tumor suppressor in gastric cancer and the inhibition of cancer cell growth may mainly be due to delaying G1-S phase transition of cells. In gastric cancer, TFF1 and GKN1 were unable to interact in protein level and cooperate in functional level.

\section{Acknowledgements}

This study was supported in part by grants from the National Natural Science Foundation of China (nos. 81072048 and 
30871145), the Natural Science Foundation of Guangdong Province (no. 7001641), the Junior Teacher Cultivation Project of Sun Yat-sen University (nos. 09ykpy22 and 10ykjc23).

\section{References}

1. Parkin DM, Bray FI and Devesa SS: Cancer burden in the year 2000. The global picture. Eur J Cancer 37 (Suppl 8): S4-S66, 2001.

2. Jemal A, Thomas A, Murray T and Thun M: Cancer statistics, 2002. CA Cancer J Clin 52: 23-47, 2002.

3. Krejs GJ: Gastric cancer: epidemiology and risk factors. Dig Dis 28: 600-603, 2010

4. Thim L: Trefoil peptides: from structure to function. Cell Mol Life Sci 53: 888-903, 1997.

5. Hoffmann W, Jagla W and Wiede A: Molecular medicine of TFF-peptides: from gut to brain. Histol Histopathol 16: 319-334, 2001.

6. Wright NA, Hoffmann W, Otto WR, Rio MC and Thim L: Rolling in the clover: trefoil factor family (TFF)-domain peptides, cell migration and cancer. FEBS Lett 408: 121-123, 1997.

7. Rio MC, Bellocq JP, Daniel JY, et al: Breast cancer-associated pS2 protein: synthesis and secretion by normal stomach mucosa Science 241: 705-708, 1988.

8. Rio MC, Chenard MP, Wolf C, et al: Induction of $\mathrm{pS} 2$ and hSP genes as markers of mucosal ulceration of the digestive tract Gastroenterology 100: 375-379, 1991.

9. Wright NA, Poulsom R, Stamp G, et al: Trefoil peptide gene expression in gastrointestinal epithelial cells in inflammatory bowel disease. Gastroenterology 104: 12-20, 1993.

10. Kjellev S: The trefoil factor family - small peptides with multiple functionalities. Cell Mol Life Sci 66: 1350-1369, 2009.

11. Henry JA, Bennett MK, Piggott NH, Levett DL, May FE and Westley BR: Expression of the pNR-2/pS2 protein in diverse human epithelial tumours. Br J Cancer 64: 677-682, 1991.

12. Machado JC, Carneiro F, Blin N and Sobrinho-Simoes M: Pattern of pS2 protein expression in premalignant and malignant lesions of gastric mucosa. Eur J Cancer Prev 5: 169-179, 1996.

13. Lefebvre O, Chenard MP, Masson R, et al: Gastric mucosa abnormalities and tumorigenesis in mice lacking the pS2 trefoil protein. Science 274: 259-262, 1996

14. Calnan DP, Westley BR, May FE, Floyd DN, Marchbank T and Playford RJ: The trefoil peptide TFF1 inhibits the growth of the human gastric adenocarcinoma cell line AGS. J Pathol 188: 312-317, 1999.

15. Bossenmeyer-Pourie C, Kannan R, Ribieras S, et al: The trefoil factor 1 participates in gastrointestinal cell differentiation by delaying G1-S phase transition and reducing apoptosis. J Cell Biol 157: 761-770, 2002.

16. Prest SJ, May FE and Westley BR: The estrogen-regulated protein, TFF1, stimulates migration of human breast cancer cells. FASEB J 16: 592-594, 2002.
17. Ather MH, Abbas F, Faruqui N, Israr M and Pervez S: Expression of $\mathrm{pS} 2$ in prostate cancer correlates with grade and Chromogranin A expression but not with stage. BMC Urol 4: 14, 2004.

18. Arumugam T, Brandt W, Ramachandran V, et al: Trefoil factor 1 stimulates both pancreatic cancer and stellate cells and increases metastasis. Pancreas 40: 815-822, 2011.

19. Sanchez-Pulido L, Devos D and Valencia A: BRICHOS: a conserved domain in proteins associated with dementia, respiratory distress and cancer. Trends Biochem Sci 27: 329-332, 2002.

20. Yoshikawa Y, Mukai H, Hino F, Asada K and Kato I: Isolation of two novel genes, down-regulated in gastric cancer. Jpn J Cancer Res 91: 459-463, 2000.

21. Toback FG, Walsh-Reitz MM, Musch MW, et al: Peptide fragments of AMP-18, a novel secreted gastric antrum mucosal protein, are mitogenic and motogenic. Am J Physiol Gastrointest Liver Physiol 285: G344-G353, 2003.

22. Walsh-Reitz MM, Huang EF, Musch MW, et al: AMP-18 protects barrier function of colonic epithelial cells: role of tight junction proteins. Am J Physiol Gastrointest Liver Physiol 289: G163-G171, 2005.

23. Baus-Loncar M, Lubka M, Pusch CM, Otto WR, Poulsom R and Blin N: Cytokine regulation of the trefoil factor family binding protein GKN2 (GDDR/TFIZ1/blottin) in human gastrointestinal epithelial cells. Cell Physiol Biochem 20: 193-204, 2007.

24. Westley BR, Griffin SM and May FE: Interaction between TFF1, a gastric tumor suppressor trefoil protein, and TFIZ1, a brichos domain-containing protein with homology to SP-C. Biochemistry 44: 7967-7975, 2005

25. Otto WR and Thim L: Trefoil factor family-interacting proteins. Cell Mol Life Sci 62: 2939-2946, 2005.

26. Kouznetsova I, Laubinger W, Kalbacher H, et al: Biosynthesis of gastrokine- 2 in the human gastric mucosa: restricted spatial expression along the antral gland axis and differential interaction with TFF1, TFF2 and mucins. Cell Physiol Biochem 20: 899-908, 2007.

27. Stolte M and Meining A: The updated Sydney system: classification and grading of gastritis as the basis of diagnosis and treatment. Can J Gastroenterol 15: 591-598, 2001.

28. Beckler AD, Roche JK, Harper JC, et al: Decreased abundance of trefoil factor 1 transcript in the majority of gastric carcinomas. Cancer 98: 2184-2191, 2003.

29. Moss SF, Lee JW, Sabo E, et al: Decreased expression of gastrokine 1 and the trefoil factor interacting protein TFIZ1/GKN2 in gastric cancer: influence of tumor histology and relationship to prognosis. Clin Cancer Res 14: 4161-4167, 2008.

30. May FE, Griffin SM and Westley BR: The trefoil factor interacting protein TFIZ1 binds the trefoil protein TFF1 preferentially in normal gastric mucosal cells but the co-expression of these proteins is deregulated in gastric cancer. Int J Biochem Cell Biol 41: 632-640, 2009

31. Rippa E, La Monica G, Allocca R, Romano MF, De Palma M and Arcari P: Overexpression of gastrokine 1 in gastric cancer cells induces fas-mediated apoptosis. J Cell Physiol 226: 2571-2578, 2011. 